\title{
MOLECULAR ANALYSIS OF XERODERMA PIGMENTOSUM GROUP A GENE
}

\author{
Kiyoji TANAKA \\ Institute for Molecular and Cellular Biology, Osaka University, \\ 1-3 Yamadaoka, Suita, Osaka 565, Japan
}

\section{Introduction}

Xeroderma pigmentosum (XP) is an autosomal recessive disease characterized by extreme sun-sensitivity of the skin and early skin cancers. Cells from XP patients are hypersensitive to killing by ultraviolet light (UV), because they have a defect in the repair of UV-induced DNA damage. Genetic complementation analysis by cell fusion has led to the identification of at least 8 genetic complementation groups, designated groups $A$ through $G$ and a variant (Table 1). Group A through G XP cells have defects in the early step of DNA excision repair. The $\mathrm{XP}$ variant was first thought to have normal excision repair and impaired postreplication repair, but recent studies have suggested that it is also defective in excision repair. Patients of complementation groups A, B, and D frequently develop various central and peripheral neurological abnormalities in addition to characteristic skin lesions, thus they are considered a neurocutaneous syndrome. We cloned a DNA repair gene that complements the defect of group A XP, the XPAC (Xeroderma Pigmentosum group $A$ Complementing) gene. Molecular analysis of the $X P A C$ gene and its product is described.

\section{Molecular cloning of the XPAC gene $e^{1,2)}$}

For isolation of the gene responsible for XP group A, plasmid pSV2gpt and genomic DNA from a mouse embryo were cotransfected into XP2OSSV cells, a group A XP cell line. Two primary UV-resistant XP transfectants were isolated from about $1.6 \times 10^{5}$ pSV2gpt-transformed XP colonies. pSV2gpt and genomic DNA from the primary transfectants were again cotransfected into XP2OSSV cells and a secondary UV-resistant XP transfectant was obtained by screening about $4.8 \times 10^{5} \mathrm{pSV} 2 \mathrm{gpt}$-transformed $\mathrm{XP}$ colonies. The secondary transfectant retained fewer mouse repetitive sequences. A mouse gene that complements the defect of XP2OSSV cells was cloned into an EMBL3 vector from the genome of a secondary transfectant. Transfections of the cloned DNA also conferred UV 
Table 1. Genetic complementation groups in xeroderma pigmentosum.

(Takebe H, Kyoto University)

\begin{tabular}{crrrrrrr}
\hline $\begin{array}{c}\text { Complementation } \\
\text { group }\end{array}$ & Japan & Europe & U.S.A. & $\begin{array}{c}\text { North } \\
\text { Africa }\end{array}$ & $\begin{array}{c}\text { China } \\
\text { Korea }\end{array}$ & $\begin{array}{c}\text { CV-UDS } \\
(\% \text { normal })\end{array}$ & $\begin{array}{c}\text { Neurological } \\
\text { abnormality }\end{array}$ \\
\cline { 2 - 6 } A & 30 & 10 & 3 & 11 & 0 & $0 \sim 5$ & + \\
B & 0 & 0 & 1 & 0 & 0 & 5 & - \\
C & 5 & 16 & 5 & 16 & 4 & $10 \sim 25$ & - \\
D & 4 & 8 & 5 & 0 & 0 & $10 \sim 30$ & + \\
E & 2 & 5 & 0 & 0 & 0 & $40 \sim 60$ & - \\
F & 11 & 2 & 0 & 0 & 1 & $10 \sim 40$ & - \\
G & 1 & 2 & 0 & 0 & 0 & 2 & + \\
Variant & 21 & 0 & 1 & 7 & 0 & $(100)$ & - \\
\hline
\end{tabular}

resistance on another group A XP cell line but not on XP cell lines of group C, D, F, or G. Northern blot analysis of poly $(A)+$ RNA with a subfragment of cloned mouse DNA repair gene as the probe revealed that an $1.0 \mathrm{~kb}$ mRNA was transcribed in the donor mouse embryo and secondary transfectant, and 1.0 and $1.3 \mathrm{~kb}$ mRNAs were transcribed in normal human cells, but none of these mRNAs was detected in three strains of group A XP cells. We then obtained human and mouse cDNAs corresponding to these mRNAs from human and mouse expression cDNA libraries. When the human $X P A C$ cDNA clone was transfected into several group A XP cell lines and lines of other XP groups, every group A XP cell line showed UV-resistance, but no UV-resistant transfectants were obtained from XP cells of other groups, confirming that the recovery of DNA repair ability by the $X P A C$ gene is specific for group A. The human and mouse XPAC genes were assigned to positions $9 \mathrm{q} 34.1$ and $4 \mathrm{C} 2$, respectively.

The human $X P A C$ cDNA has a single large open reading frame predicted to encode 273 amino acid residues. The deduced molecular weight was about 31 $\mathrm{kDa}$. No proteins with extensive sequence similarity with the XPAC protein were found in a search of the National Biomedical Research Foundation database. A mouse XPAC protein was $95 \%$ similar to a human XPAC protein. A computer search of the secondary structure using the program of Chou and Fasman revealed several $\alpha$-helix-turn-helix structures in the XPAC protein. In addition, the XPAC protein contains zinc-finger motifs, $\mathrm{CX}_{2} \mathrm{CX}_{3} \mathrm{FX}_{4} \mathrm{LX}_{2} \mathrm{HX}_{6} \mathrm{CX}_{2} \mathrm{C}$ at amino acid positions 105-129 and $\mathrm{HXHX}_{10} \mathrm{CX}_{2} \mathrm{C}$ at amino acid positions 242-264. This finding strongly suggests that the XPAC protein is a DNA-binding protein (Fig. 1).

\section{Identification and characterization of the cellular XPAC protein ${ }^{3)}$}

In order to identify and characterize the XPAC protein, we produced a recombinant XPAC protein in E. coli using pET-3 expression vector. The expected 


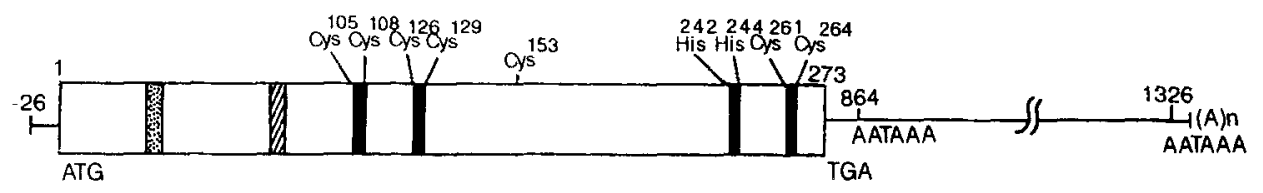

Zinc-finger motif

Nuclear localization signal

E-cluster

Fig. 1. Structure of XPAC protein.

protein was a fusion protein with 14 extra amino acids of bacteriophage T7 major capsid protein. Microinjection of a recombinant XPAC protein into group A XP cells resulted in the restoration of normal level of UV-induced unscheduled DNA synthesis or 4-nitroquinoline 1-oxide (4NQO).

Rabbits were immunized with the purified recombinant XPAC protein to obtain anti XPAC protein antiserum. By immunoprecipitation and SDS-PAGE analysis, two proteins with relative molecular mass of 40 and $38 \mathrm{kDa}$ were specifically detected in the preparation of normal human cells, but no specific proteins were detected in that of group A XP cells (XP2OSSV) (Fig. 2). pcD2h19 (SV40 promotor-driven human XPAC cDNA expression plasmid)-transfected XP2OSSV cells also showed the same two bands of 40 and $38 \mathrm{kDa}$ as normal human cells. By western blot analysis, the 40 and $38 \mathrm{kDa}$ proteins were also detected in normal human cells. From these results, we conclude that the proteins migrating at positions corresponding to molecular masses of 40 and $38 \mathrm{kDa}$ were XPAC proteins. The immunofluorescence study revealed that the XPAC protein was mainly localized in the nucleus in normal human cells (Fig. 4), but no staining of the XPAC protein was detected in group A XP cells (XP2OSSV). We then examined whether anti XPAC antibody inhibited the DNA repair activity in HeLa cell ext1act. When a HeLa cell extract $(5 \mathrm{mg} / \mathrm{ml})$ was injected into group A XP cells (XP12BE), UVinduced unscheduled DNA synthesis was restored to the normal level. Preincubation of a HeLa cell extract with anti XPAC protein antibody completely abolished its complementing activity to group A XP cells. These results indicate that the XPAC protein is the only protein defective in group A XP cells.

For further characterization of the XPAC protein, we synthesized it by in vitro translation of in vitro transcripts from the cloned XPAC cDNA. The in vitro translated proteins had identical electrophoretic mobilities to those of in vivo-labeled proteins, and the two bands were recognized by anti-XPAC protein serum. Therefore, the possibility that the alternative splicing results in two proteins was excluded.

In a pulse-chase experiments in WI38VA13 cells in which the cells were pulselabeled for 10 min and chased in Dulbecco's modified Eagle's medium for various 


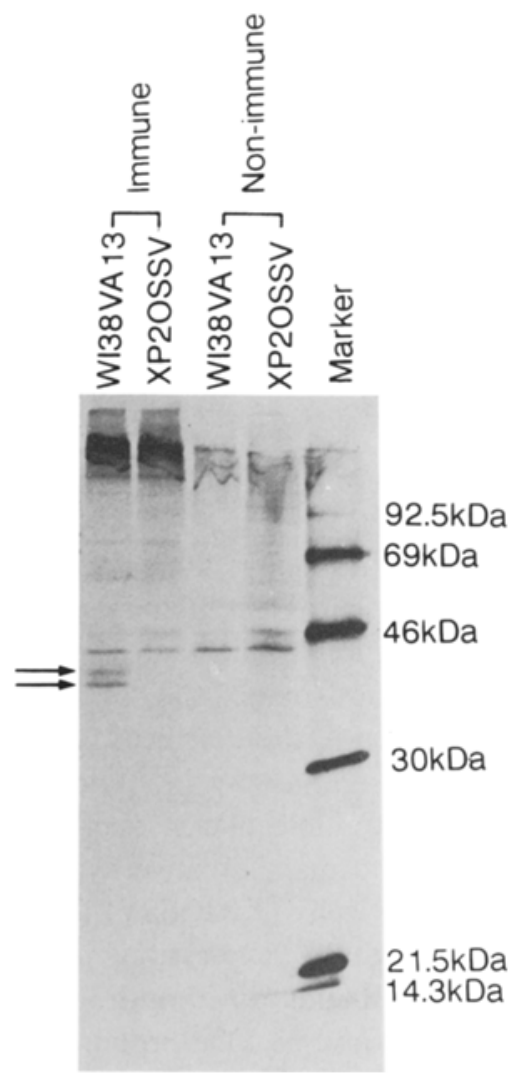

Fig. 2. Immunoprecipitation of XPAC protein. Normal human cells (WI38VA13) and group A XP cells (XP2OSSV) were metabolically labeled, solubilized, immunoprecipitated with preimmune serum or anti XPAC protein serum, and analyzed by SDS-PAGE and fluorography.

times, the ratio of the intensities of the 40 and $38 \mathrm{kDa}$ bands remained constant from $0 \mathrm{~min}$ to $4 \mathrm{~h}$ of chase.

If the translated proteins starting from the second methionine at amino acid position 37 , the difference in sizes of the proteins starting at the first and second methionines should be about $4 \mathrm{kDa}$. When a mutant cDNA construct that contained the second methionine as the initial translation site was transfected into group A XP (XP2OSSV) cells, we detected two expected sizes of bands with molecular masses of 36 and $34 \mathrm{kDa}$, suggesting that the two bands of XPAC protein were not due to double translation initiation.

When WI38VA13 cells and XP2OSSV cells were metabolically labeled with ${ }^{[2} \mathrm{P}_{3} \mathrm{H}_{3} \mathrm{PO}_{4}$, solubilized, and immunoprecipitated with anti XPAC serum, no ${ }^{32} \mathrm{P}$ incorporation into the two bands of XPAC protein was detected. Sometimes nuclear proteins have $O$-linked $N$-acetylglucosamine (GlcNAc) monosaccharides 
residues. To test whether the XPAC proteins also have GlcNAc residues, we translated them in vitro and loaded them on a wheat germ agglutinin-agarose column. The 40 and $38 \mathrm{kDa}$ proteins detected in the flow through fraction but not in the eluate with 0.3 M GICNAc. Thus, we obtained no evidence that the XPAC protein was posttranslationally modified or processed. We do not know the exact mechanism of production of two forms of the XPAC protein in normal human cells. The possibilities that the $38 \mathrm{kDa}$ protein is a degradation product of the $40 \mathrm{kDa}$ protein and that the $40 \mathrm{kDa}$ protein is a posttranslationally modified form of the $38 \mathrm{kDa}$ protein are not yet be excluded.

We examined whether synthesis of the XPAC protein increased after UVirradiation. For this, WI38VA13 cells were irradiated at up to $4 \mathrm{~J} / \mathrm{m}^{2}$, and 2,4 , and $8 \mathrm{~h}$ later, control and UV-irradiated cells were labeled with $\left[{ }^{35} \mathrm{~S}\right]$ methionine for $30 \mathrm{~min}$, solubilized, and processed for immunoprecipitation, SDS-PAGE, and fluorography. The de novo synthesis of XPAC protein was not changed, 2,4 , and $8 \mathrm{~h}$ after UV-irradiation.

\section{DNA binding ability of the recombinant $X P A C$ protein}

(Asahina $\mathrm{H}$ et al., manuscript in preparation)

Since the XPAC protein has zinc-finger motifs, a DNA binding activity of the XPAC protein was examined by gel retardation and filter binding assays. The 80 mer oligonucleotides with or without one pyrimidine dimer or UV $\left(6 \mathrm{KJ} / \mathrm{m}^{2}\right)$ irradiated or unirradiated 65 mer oligonucleotides with consecutive thymine residues were synthesized, labeled by $\left[\gamma^{32}{ }^{32}\right]$ ATP and used as probes for gel retardation and filter binding assays. The probe mixed with the XPAC protein was retarded in the gel, while the extract from $E$. coli transfected with $\mathrm{pET}-3$ vector did not cause any retardation. This result indicates that the XPAC protein has a DNA binding activity, but has no specificity to pyrimidine dimer containing oligonucleotides or UV-damaged oligonucleotide. The quantitative analysis of the DNA binding activity by filter binding assay also revealed that the XPAC protein has no specificity to UV-damaged oligonucleotides. On the other hand, when the bateriophage M13 DNA was used as a probe instead of oligonucleotides, the filter binding assay revealed that more XPAC protein bound to UV-irradiated $\left(6 \mathrm{KJ} / \mathrm{m}^{2}\right)$ DNA than to nonirradiated DNA. These results indicate that the XPAC protein can recognize DNA damages which reside in the long chain of DNA and is involved in the recognition step of the damaged sites in DNA excision repair process.

\section{Mutational analysis of the structure and function of the XPAC protein ${ }^{4)}$}

The XPAC protein involved in the DNA excision repair pathway contains zinc-finger motifs and is localized in the nucleus of normal human cells. For detailed structural and functional analyses of the XPAC protein, various mutant $X P A C$ cDNAs were constructed by site-directed mutagenesis, transfected into group A XP cells (XP12ROSV) and expressed by CMV enhancer and chicken $\beta$ actin promotor. Permanent cell lines expressing mutant proteins were isolated. DNA 
repair ability of these mutant cDNAs were assessed by measuring the UV-survival of these transfectants.

The XPAC protein has seven cysteines. Four of them $(105,108,126$, and 129 amino acid residues) are supposed to form a $\mathrm{C}_{4}$ type zinc-finger motif, and two (261 and 264 amino acid residues) are supposed to form a $\mathrm{H}_{2} \mathrm{C}_{2}$ type zinc-finger motif. In order to examine the importance of these cysteines on the DNA repair function of the XPAC protein, cysteine residues were replaced by serine. Replacement of any cysteine in the $\mathrm{C}_{4}$ zinc-finger motif by serine severely affected the DNA repair activity. Replacement of each one of the cysteines in the $\mathrm{H}_{2} \mathrm{C}_{2}$ type zincfinger motif by serine also caused partial loss of DNA repair activity. Replacement of cysteine in the $\mathrm{C}_{4}$ type zinc-finger motif by phenylalanine caused complete loss of DNA repair activity of the XPAC protein. On the other hand, replacement of the solitary cysteine (153 amino acid residue) by serine did not affect any DNA repair activity (Fig. 3).

We also made the mutant $X P A C$ cDNAs with $\mathrm{N}$-terminal or C-terminal deletions. $\triangle \mathrm{N} 36$ (mutant with $\mathrm{N}$-terminal 36 amino acids deletion)- and $\triangle \mathrm{N} 58$-transfected group A XP cells showed complete DNA repair activity, whereas $\Delta \mathrm{N} 97$ cells

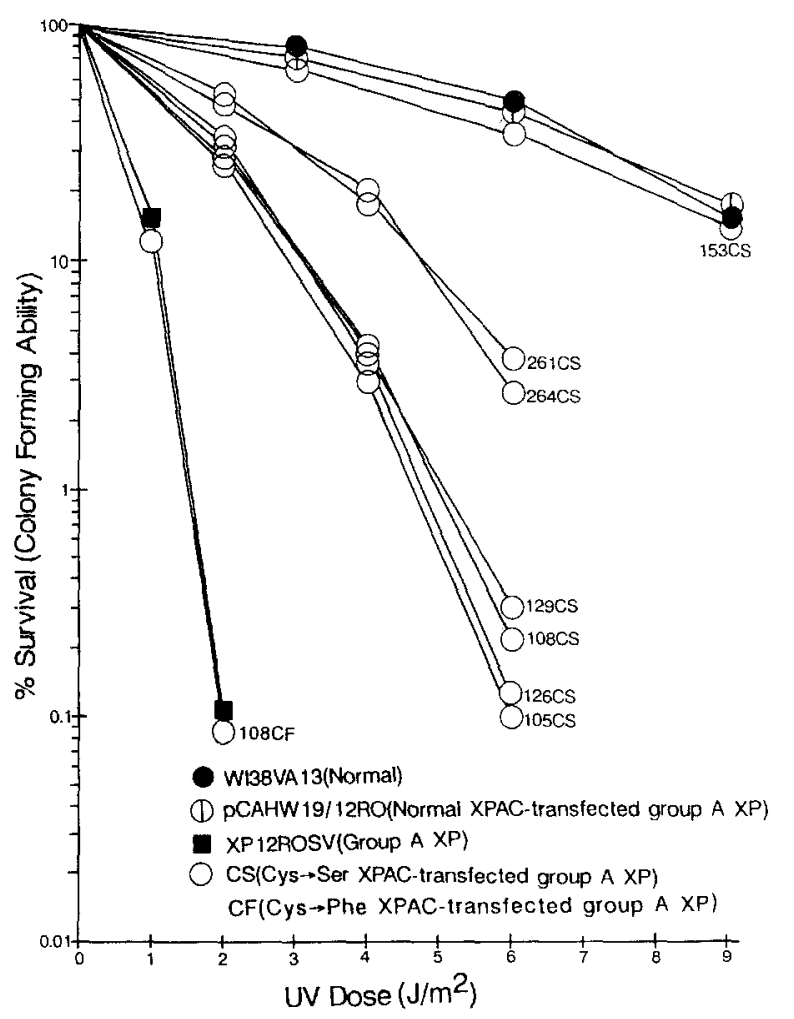

Fig. 3. UV-survivals of mutant XPAC cDNA-transfected XP cells. 
showed total loss of activity. Similarly, ANLS (nuclear localization signal) cells, the deletion mutant with 30-42 amino acids, showed complete repair activity. On the other hand, C-terminal deletions and exon 2-5 deletions did not affect nuclear localization, but completely abolished DNA repair activity. These results indicate that exon 1 containing the putative NLS is not essential for DNA repair function, but all exons except exon 1 are important for DNA repair. The XPAC protein without NLS can be located into nucleus by passive diffusion through nuclear pore and these XPAC protein in the nucleus is sufficient to restore DNA repair defect in group A XP cells.

The basic amino acid sequence Arg-Lys-Arg-Gln-Arg-Ala-Leu-Met-Leu-ArgGln-Ala-Arg at amino acids $30-42$ in exon 1 is the putative NLS of the XPAC protein. To confirm this, we made mutant $X P A C$ cDNA which lacks this region by site-directed mutagenesis. The wild type or mutant $X P A C$ cDNA was transfected and expressed in group A XP cells in which no XPAC protein was detected, and the transfectants were stained with anti XPAC protein antibody. In the wild type cDNA-transfected clone, the nuclei were clearly stained, while in the mutant cDNAtransfected clone, the intensity of fluorescence in the nucleus and cytoplasm of the cells stained with anti XPAC antiserum was indistinguishable (Fig. 4). These results indicate that the NLS is located in the amino acids $30-42$.

We examined the wild type and mutant XPAC proteins in the transfectants by immunoprecipitation analysis with anti XPAC serum. Two major bands with molecular masses of 40 and $38 \mathrm{kDa}$ were precipitated in wild type $X P A C$ cDNA transfected cells. The XPAC proteins were expressed at about 5 times higher
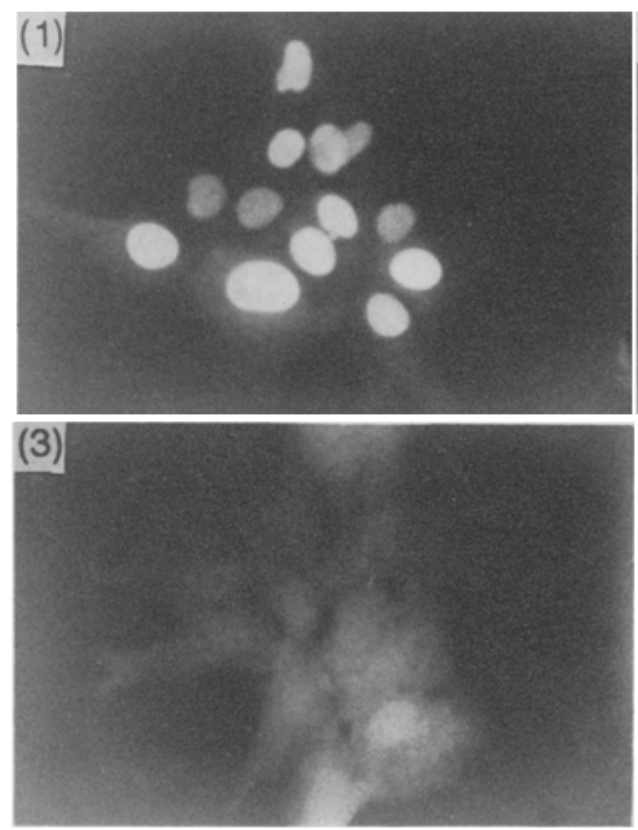

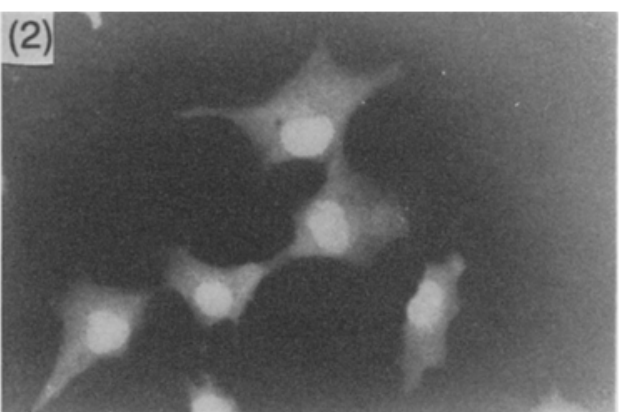

Fig. 4. Intracellular localization of wild type $\triangle$ NLS mutant XPAC proteins in various transfectants. (1) Wild type $X P A C$ cDNA-transfected XP12ROSV cells. (2) $\triangle$ NLS ( ${ }^{37}$ Met $-{ }^{58}$ Gly deficient) $X P A C$ cDNA-transfected XP12ROSV cells. (3) $\triangle \mathrm{NLS}\left({ }^{30} \mathrm{Arg}-{ }^{42} \mathrm{Arg}\right.$ deficient) $X P A C$ cDNA-transfected XP12ROSV cells. Cells were treated with anti XPAC serum as the first antibody, and then with FITC-conjugated goat anti rabbit IgG as the second. 
level than endogenous XPAC proteins in normal human cells. N-terminally deleted mutant XPAC proteins, C-terminally deleted mutant XPAC proteins, and internally deleted mutant XPAC proteins were detected at the positions of their predicted sizes in lysates of the transfectants. Transfection of C-terminal 70 amino acids deletion mutant of the $X P A C$ cDNA into group A XP cells resulted in the production of only one band of truncated XPAC proteins, while C-terminal 48 amino acids deletion mutant of the XPAC cDNA generated two bands of truncated XPAC proteins, suggesting that the regions from 48 to 70 amino acids are involved in the two forms of the XPAC proteins.

${ }^{108}$ Cysteine to serine or phenylalanine mutant $X P A C$ cDNA was inserted into pET-3 vector and expressed in $E$. coli and each of these mutant XPAC proteins were produced in $E$, coli and its DNA binding activity was examined by gel retardation assay. ${ }^{108}$ Cysteine to serine mutant XPAC protein bound to DNA a very little, while ${ }^{108}$ cysteine to phenylalanine mutant did not bind to DNA at all. These results indicate that the zinc-finger motif which is involved in DNA binding has an important role in DNA repair activity of the XPAC protein.

\section{Molecular cloning and characterization of XPAC gene homologs ${ }^{5)}$}

We cloned homologs of the human $X P A C$ gene from chicken, Xenopus laevis and Drosophila melanogaster. A comparison of the amino acid sequences of these homologs with that of the human XPAC protein revealed that in the N-terminal domain there are only two conserved regions, one of which functions as the nuclear localization signal, whereas the C-terminal region is highly conserved, the frequency of identical amino acids in all four XPAC proteins being $50 \%$, and all the cysteine residues predicted to form zinc-finger motifs are conserved (Fig. 5). These results strongly suggest that the $\mathrm{C}$-terminal region including zinc-finger motifs plays an important role in the function of these proteins. It has been reported that $R A D 14$ is a yeast homolog of the human $X P A C$ gene. ${ }^{6)}$ The zinc-finger motifs are conserved in yeast, and the homology is extensive in the C-terminal portion of the two proteins, sharing about $30 \%$ identical residues. These results are consistent well with those by our in vitro mutagenesis experiments.

\section{Proteins associated with the XPAC protein}

(Iwai $\mathrm{K}$ et al., manuscript in preparation)

All the complementation groups of XP cells have defects in the early step(s) of DNA excision repair, suggesting that at least $7(8)$ different genes are involved in the early step(s) of DNA excision repair. It is possible that XP proteins may associate with each other or associate with other proteins which are involved in DNA excision repair, cell cycle, DNA replication or transcription, and form multiprotein complex during DNA excision repair process. In order to examine the possibility that the XPAC protein may associate with some proteins, the XPAC proteins were mixed with cellular extracts from XP2OSSV cells. The mixture was then overlayed onto $5-20 \%$ glycerol gradient, spun at $48 \mathrm{~K} \mathrm{rpm}$ for $1 \mathrm{~h}$ and fractionated. 
(1)

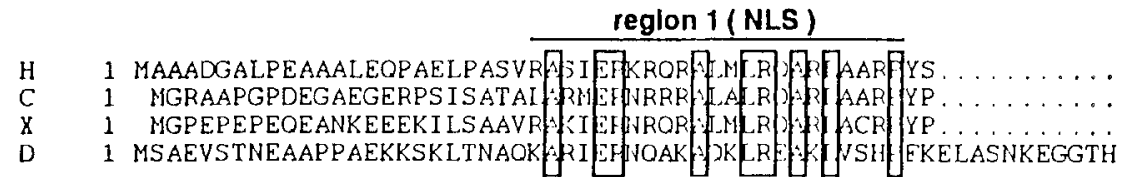

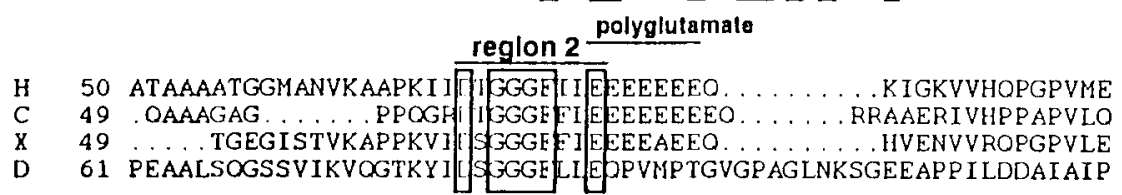

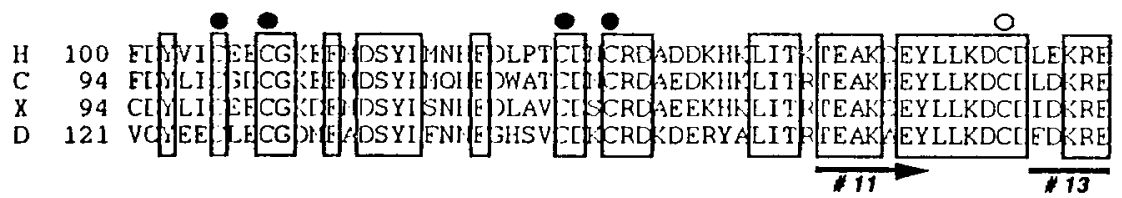
$\begin{array}{ll}\mathrm{H} & 160 \\ \mathrm{C} & 154 \\ \mathrm{X} & 154 \\ \mathrm{D} & 181 \\ \mathrm{E}\end{array}$

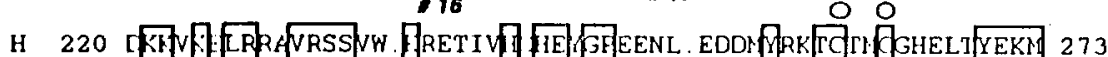

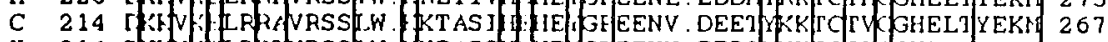

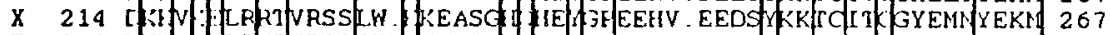

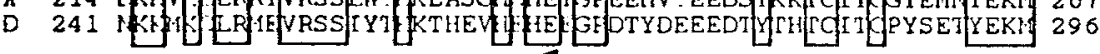

(2)

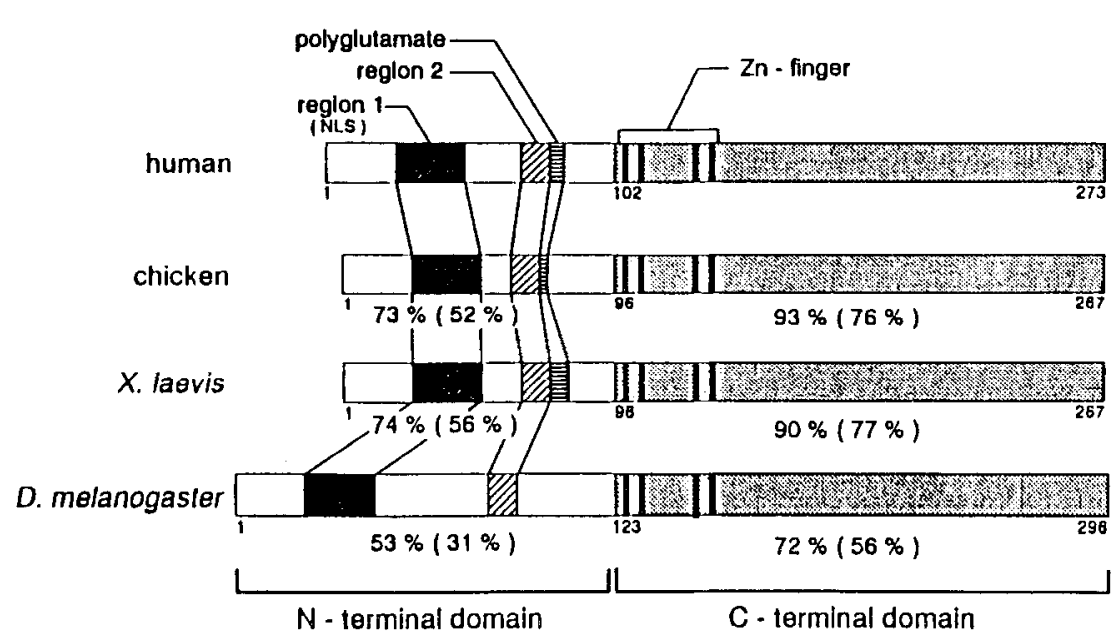

Fig. 5. Amino acid alignment of the XPAC proteins of human $(\mathrm{H})$, chicken $(\mathrm{C}), X$. laevis (X) and $D$. melanogaster (D). (1) Amino acids that are identical in all four proteins are boxed. Circles indicate 7 cysteine residues conserved in all four proteins. (2) Diagram of the domains and regions revealed by sequence comparison. Numbers indicate percent similarity to the human XPAC protein.

Vol. 38, No. 1, 1993 
The XPAC proteins in each fraction were detected by western blot analysis with anti XPAC protein antiserum. The XPAC proteins mixed with cell extract from XP2OSSV cells were sedimented into heavier fractions than the free XPAC proteins. We also produced glutathione $S$-transferase(GST)-XPAC fusion proteins in E. coli. GST can bind to glutathione Sepharose $4 \mathrm{~B}$ beads, and therefore, GST-XPAC fusion proteins can be immobilized to glutathione Sepharose $4 \mathrm{~B}$ beads. The cell extract from XP2OSSV cells which have been metabolically labeled with $\left[{ }^{35}\right.$ S]methionine were mixed with the GST-XPAC Sepharose beads. After washing the beads with the buffer, proteins associated with the GST-XPAC fusion proteins were analyzed by SDS-PAGE and autoradiography. Although several proteins were bound with GST beads, more proteins were bound with GST-XPAC beads. The associations of some of these proteins were inhibited by pretreatment of the beads with anti-XPAC protein antiserum, or addition of synthetic peptides which contain a putative pocket domain of the XPAC protein. Moreover, associations of some of these proteins were increased in the presence of UV-irradiated DNA. These results indicate that the XPAC protein may associate with several proteins in vivo in the DNA excision repair process.

\section{Molecular basis of group A xeroderma pigmentosum ${ }^{7-10}$}

Southern blot analysis of genomic DNA from different group A patients with $X P A C \mathrm{cDNA}$ as a probe revealed that there were no gross genomic rearrangements of the $X P A C$ gene in any group A XP patients tested. On the other hand, northern blot analysis of poly $(\mathrm{A})^{+} \mathrm{RNA}$ revealed $X P A C \mathrm{~m}$.RNAs of about 1.3 and $1.0 \mathrm{~kb}$ in cells of group $B$ through $G$ and a variant $X P$ and in normal human cells, but abnormal XPAC mRNA in almost all group A patients. Almost all the Japanese patients showed identical abnormalities in XPAC mRNAs, indicating a common origin of mutation of the $X P A C$ gene.

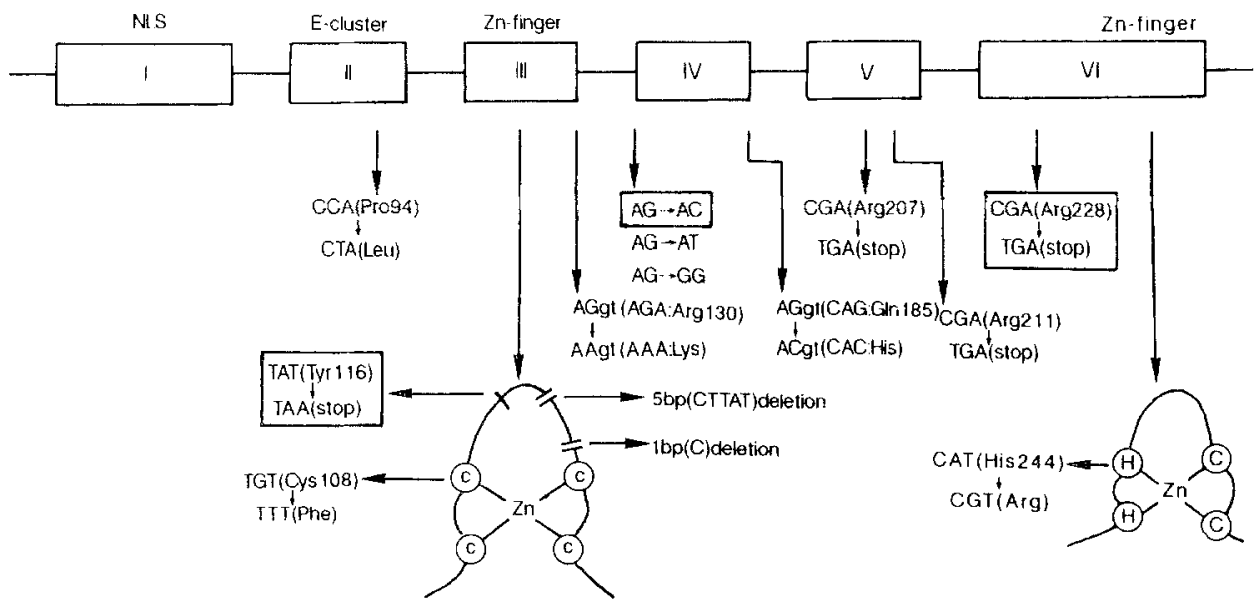

Fig. 6. Mutations of the XPAC gene in XP group A patients. I-VI indicate exons of the XPAC gene. Mutations found in Japanese patients are boxed. 
To examine the molecular basis of group A XP, the human XPAC gene was cloned from normal and group A XP cells. The human $X P A C$ gene is about 25 $\mathrm{kb}$ long and is split into six exons. Comparison of the nucleotide sequences of multiple M13 clones of the XPAC gene from group A XP cells with those of normal human cells revealed that there are three kinds of mutations of the $X P A C$ gene in Japanese group A XP patients (Fig. 6). One was a $\mathrm{G}$ to $\mathrm{C}$ transversion at the 3' splice acceptor site of intron 3, which altered the obligatory AG acceptor dinucleotides to AC, creating two abnormally spliced mRNA forms. The larger form is identical to normal mRNA except for a dinucleotides deletion at the $5^{\prime}$ end of exon 4. This deletion results in a frameshift with premature translation termination in exon 4. The smaller form has a deletion of the entire exon 3 and the dinucleotides at the $5^{\prime}$ end of exon 4 . This $\mathrm{G}$ to $\mathrm{C}$ single base substitution creates a new cleavage site for A1wNI restriction endonuclease (Fig. 7). Analysis of A1wNI restriction fragment length polymorphism (RFLP) showed a high frequency $(80-90 \%)$ of this splicing mutation in Japanese group A XP patients. Sixteen out of 21 Japanese group A XP patients were found to be homozygous for this splicing mutation. Second mutation was a $C$ to $T$ transition in exon 6 altering

A

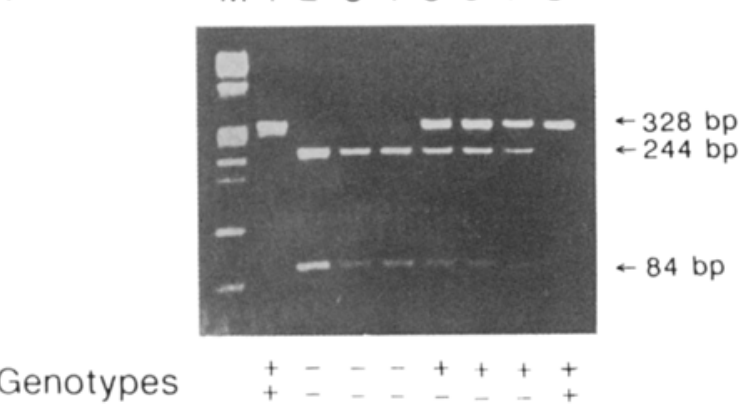

B

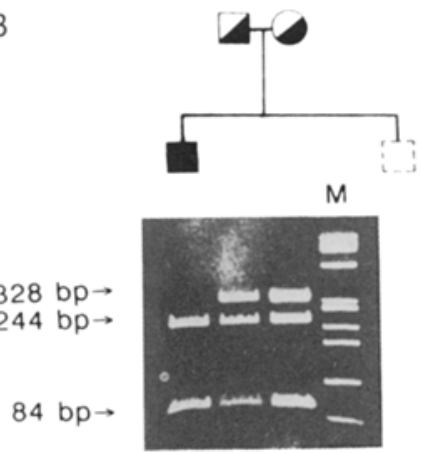

C

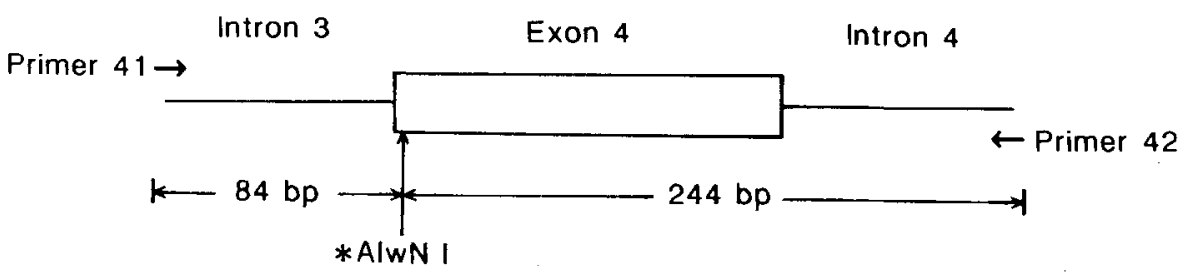

Fig. 7. A1wNI RFLP analysis of amplified XPAC DNAs. (A) Aliquots of PCR products were digested with A1WNI and then analyzed by $8 \%$ polyacrylamide gel electrophoresis. Lanes 1 and 8 , normal human cells; lanes 2-4, group A XP cells homozygous for the mutation; lanes $5-7$, group A XP cells heterozygous for the mutation. (B) Pedigree of XP84TO analyzed by A1wNI RFLP. (C) Diagram of the positions for PCR primers and the location of the new A1wNI site (asterisk) generated by the single base substitution. 
Arg codon (CGA) to a nonsense codon (TGA). One of the Japanese group A XP patients (XP39OS) who showed mild skin symptoms with no skin tumors or neurological abnormalities was homozygous for this mutation. In XP39OS cells, the amount of XPAC mRNA was similar to that of normal cells, and it was expected that the truncated XPAC protein lacking the C-terminal 46 amino acids was produced in XP39OS cells. On SDS-PAGE analysis after immunoprecipitation with anti-XPAC serum, two bands of protein of 35 and $33 \mathrm{kDa}$ were detected in the preparation from XP39OS cells. However, densitometric measurement showed that the amount of XPAC protein in XP39OS cells was about one-fifth of that in normal human cells. The third mutation was a $\mathrm{T}$ to $\mathrm{A}$ transversion in exon 3 altering Tyr codon (TAT) to a nonsense codon (TAA). Of 21 unrelated Japanese group A XP patients, 2 with severe clinical symptoms had this mutant allele. These two nonsense mutations also created new cleavage sites for restriction endonucleases $H p h \mathbf{I}$ and $M s e I$, respectively. These mutations found in Japanese group A XP patients were not detected in non-Japanese group A XP patients. We identified three mutations located in a zinc-finger consensus sequences (nucleotide 313-387) of the XPAC gene in 2 Caucasian patients XP1CA and XP1PD who had typical symptoms of group A XP (Fig. 6). One was a $C$ deletion at nucleotide 374. Patient XP1CA was homozygous for this mutation. The second mutation was a 5 bp deletion (CTTAT) at nucleotide 349-353. The third mutation was a $\mathrm{G}$ to $\mathrm{T}$ transversion at nucleotide 323 , which alters the ${ }^{108} \mathrm{Cys}$ codon (TGT) to a Phe codon (TTT). Patient XP1PD was a compound heterozygote for the $5 \mathrm{bp}$ deletion and the missense mutation. Both deletions introduce frameshifts with premature translation terminations resulting in instability of the $X P A C \mathrm{~m}$ RNA and disruption of the C4 type zinc-finger domain of the XPAC protein. These results indicate that the zinc-finger motifs are important for DNA repair function of the XPAC protein in vivo.

\section{Establishment of XP mouse deficient in the XPAC gene}

(Yuba $\mathrm{S}$ et al., manuscript in preparation)

In order to understand the pathogenesis of cancer-proneness and neurological abnormalities in group A XP patients, the establishment of the model mouse of group A XP by gene targeting in mouse embryonic stem (ES) cells is indispensable. To disrupt one allele of the $X P A C$ gene, a replacement vector was constructed with the neo gene. The $X P A C$-neo sequence are flanked by the $H S V$-tk gene to enrich homologous integration events according to the positive-negative selection protocol. The vector was introduced into ES cell line F1/1 by electroporation. DNA was extracted from the G418 and GANC resistant colonies. Homologous integrants were distinguished from random integrants by PCR analysis and genomic Southern blot analysis. The frequency of targeting events, expressed as the number of homologous recombinants over the number of stably transfected cells, was about 7 in 37. To test the phenotype of recessive mutation in the future XP model mouse, we sequentially inactivated the remaining wild allele of the $X P A C$ gene in the 
heterozygous line by secondary gene targeting. For construction of a secondary targeting vector, the neo cassette in the primary targeting vector was replaced for a hyg cassette. This targeting vector was introduced into the heterozygous ES cell line, and the cells were selected with G418, hygromycin B and GANC. The frequency of secondary targeting events was 1 in 218 by positive-negative selection for G418, hygromycin B and GANC. The insertion of hyg or neo gene into exon 4 of the XPAC gene would abolish the normal expression of the gene. To examine this anticipation, northern blot analysis was performed with the $X P A C$ cDNA probe. An aberrant mRNA could be produced from the each inactivated allele. The normal transcript of the XPAC gene was seen in the wild type and heterologous ES cell line but not in the homozygous cell line. The heterozygote showed about $50 \%$ decrease in the amount of the normal XPAC mRNA. Furthermore, the homozygote carrying mutated allele(s) expressed several extended mRNAs. One of these mRNAs, $c a 18 \mathrm{~S}$, might be transcribed over the inserted neo or hyg cassette and the others might be aberrantly spliced messages. The mutant XPAC protein by these aberrant mRNAs, if any, might not have any DNA repair activity. To study the DNA repair ability of these cell lines, their survivals after UV-irradiation were determined. The relative sensitivity to UV of the heterozygote was the same level as that of the wild type line, while the homozygote was more sensitive to the lethal effects of UV than the wild type or heterozygote. These results encouraged us to continue a trial into the production of the group A XP model mouse with UV-sensitivity. We recently obtained germ line chimera by aggregating the heterozygous ES cells with 8 cells embryo, and also heterozygous mice. The homozygous mice were then obtained by mating the heterozygous mice. XPAC deficient mouse should be useful not only for analyzing pathogenesis of XP but also for scientific trial of gene therapy of XP.

\section{REFERENCES}

1. Tanaka K, Satokata I, Ogita Z, Uchida T, Okada Y (1989): Molecular cloning of a mouse DNA repair gene that complements the defect of group-A xeroderma pigmentosum. Proc Natl Acad Sci USA 86: 5512--5516

2. Tanaka K, Miura N, Satokata I et al. (1990): Analysis of a human DNA excision repair gene involved in group A xeroderma pigmentosum and containing a zinc-finger domain. Nature 348: 736

3. Miura N, Miyamoto I, Asahina H, Satokata I, Tanaka K, Okada Y (1991): Identification and characterization of XPAC protein, the gene product of the human XPAC (Xeroderma Pigmentosum Group A Complementing) gene. J Biol Chem 266: 19786-19789

4. Miyamoto I, Miura N, Niwa H, Miyazaki J, Tanaka K. (1992): Mutational analysis of the structure and function of the xeroderma pigmentosum group A complementing protein. $\mathrm{J}$ Biol Chem 267: 12182-12187

5. Shimamoto T, Kohno K, Tanaka K, Okada Y (1991): Molecular cloning of human $X P A C$ gene homologs from chicken, Xenopus laevis and Drosophila melanogaster. Biochem Biophys Res Commun 181: 1231-1237 
6. Bankmann M, Prakash L, Prakash S (1992): Yeast RAD14 and human xeroderma pigmentosum group A DNA-repair genes encode homologous proteins. Nature 355: 555-558

7. Satokata I, Tanaka K, Miura N, Miyamoto I, Satoh Y, Kondo S, Okada Y. (1990): Characterization of a splicing mutation in group A xeroderma pigmentosum. Proc Natl Acad Sci USA 87: $9908-9912$

8. Satokata I, Tanaka K, Miura $\mathrm{N}$ et al. (1992): Three nonsense mutations responsible for group A xeroderma pigmentosum. Mutat Res 273: 193-202

9. Satokata I, Tanaka K, Yuba S, Okada Y (1992): Identification of splicing mutations of the last nucleotides of exons, a nonsense mutation, and a missense mutation of the XPAC gene as causes of group A xeroderma pigmentosum. Mutat Res 273: 203-212

10. Satokata I, Tanaka K, Okada Y (1992): Molecular basis of group A xeroderma pigmentosum: a missense mutation and two deletions located in a zinc-finger consensus sequence of the $X$ $P A C$ gene. Hum Genet 88: 603-607 